\title{
Effect of the Microcrack Inclination Angle on Crack Propagation Behavior of TiAl Alloy
}

\author{
R. C. Feng, ${ }^{a, b}$ J. T. Lu, ${ }^{a, b, 1}$ H. Y. Li, ${ }^{a, b}$ H. Cao, ${ }^{a, b}$ and Z. Y. Rui ${ }^{a b b}$ \\ ${ }^{a}$ Key Laboratory of Digital Manufacturing Technology and Application, the Ministry of Education, \\ Lanzhou University of Technology, Lanzhou, China \\ ${ }^{\mathrm{b}}$ School of Mechanical and Electronical Engineering, Lanzhou University of Technology, Lanzhou, \\ China \\ ${ }^{1}$ Lujiant@163.com
}

Molecular dynamics simulation was employed to make the model of crack propagation for singlecrystal $\gamma$-TiAl alloy. The effects of the angle between a microcrack and loading direction on crack propagation were studied through the analysis of the atomic configurations and stress-strain curves in the paper. The results show that the time for the emission of first dislocation and the yield stress value decrease with the angle. The crack propagation occurs by the submain crack mechanism, which becomes more obvious as the angle increases and the number of stacking faults and stair-rod dislocations decreases.

Keywords: microcrack, $\gamma$-TiAl alloy, crack propagation, molecular dynamics.

Introduction. Because of low density, high specific stiffness, good high temperature strength, and creep resistance, $\gamma$-TiAl alloys are widely used in the aerospace industry $[1$, 2]. However, life of structural components is controlled by crack initiation and propagation under loading conditions. A large number of experiments showed that crack initiation greatly reduced the mechanical properties of material and directly affected the residual life, while many scholars focus their studies on the crack propagation stage [3]. However, due to the development of the computer technologies, the molecular dynamics method has been introduced as an important research tool in the materials science field [4].

At present, scholars have done a lot of research on crack propagation using the molecular dynamics method. Terentyev et al. [5] investigated the effects of the strain rate, temperature and the crack shape on crack propagation behavior of bcc and fcc metals, and the conclusion that bec metals are more prone to brittle fracture rather than ductile one. For fcc metals, when dislocations are emitted, the crack tip is blunted. Zhou et al. [6] studied the effects of grain boundaries on crack propagation behavior of bicrystal magnesium, and found that as the grain boundary angle increases, crack experiences the sharpeningblunting-sharpening cyclic variations. Karimi et al. [7] analyzed the crack propagation behavior of single-crystal nickel with defects using the molecular dynamics method. Yan et al. [8] investigated the effects of temperature on crack propagation mechanism of singlecrystal aluminum with the edge crack or center crack, and concluded that the edge crack yield stress is higher than that of the central crack at low temperature, but with an increase in temperature, the result is contrary. Guo [9] simulated the mode I crack propagation process of single-crystal Si and Al using molecular dynamics method, and showed that crack does not propagate by the cleavage mechanism, but becomes blunted because of dislocation emission from the crack tip, and a disordered region is formed due to the pile-up of disordered atoms at the crack tip. The crack propagates by means of the driving force moving the disordered regions. Rafii-Tabar et al. [10] and Mousazadeh and Dehghani [11] studied the effects of impurities on mode I crack propagation via the molecular dynamics method. Tang et al. [12] generalized a previous study of the effects of the model size, strain rate and temperature on crack propagation, and the results showed that with the 
increase of the model, the yield stress decreases, in addition to the minimal model. Strain rate and temperature increase also reduce the yield stress, while the elastic modulus is not affected. Researchers [13-15] studied the effect of temperature on crack propagation.

The above studies were focused on the effect of strain rate, temperature and the shape of crack on crack propagation. However, the existing crack modes can vary, as well as the angles between microcracks and loading direction. Therefore, the effects of the angle between microcrack and the loading direction on crack propagation are investigated in this paper, and the angle of 0,45 , and $90^{\circ}$ are chosen to study the mechanical properties of single-crystal $\gamma$-TiAl alloys. The model is shown in Fig. 1.

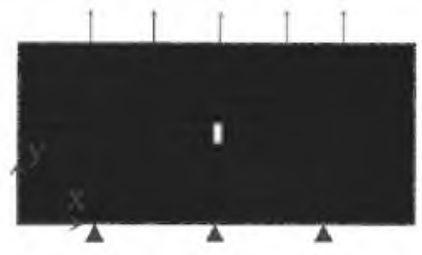

a

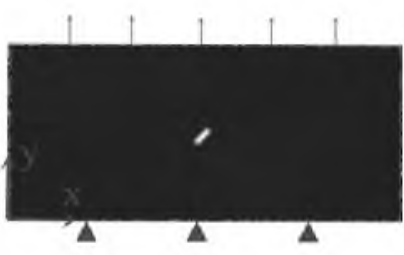

b

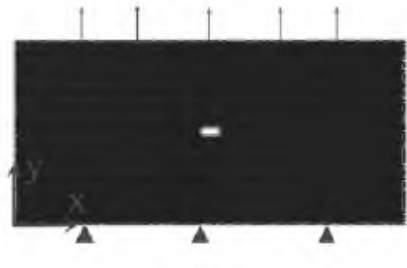

c

Fig. 1. Schematic diagram of the model with various crack inclination angles: 0 (a), 45 (b), and $90^{\circ}$ (c).

1. Method. $\gamma$-TiAl alloy has face-center tetragonal (FCT) structure with $\mathrm{L} 1_{0}$ structure, as shown in Fig. 2 [16]. The crystal orientation [100], [010], and [001] corresponds to $x, y$, and $z$ axes, respectively.

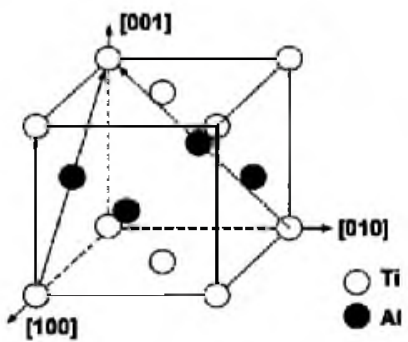

Fig. 2. Atomic configurations of $\gamma$-TiAl unit cell.

To observe the crack propagation behavior, the model that contains as many atoms as possible. To avoid excessive calculation, the model should be not too large. Therefore, the size of the model is $100 a \times 45 a \times 10 c$, where $a=0.405 \mathrm{~nm}$ and $c / a=1.045$. The initial pre-crack was introduced by removing the atoms. The initial crack length is $4 a$. To achieve plane strain condition, the periodic boundary condition was assigned in the $z$ direction. The border atomic layers of $y$ direction were fixed by LAMMPS [17] programming method. With the purpose of eliminate the stress oscillation, the loading was carried out by applying velocity along the $y$ direction. Atomic surface layers at the upper boundary were fixed and applied mode I loading. The thickness of the atomic surface layers should be larger than atoms cut-off distance [18]. For the sake of alleviate the boundary effects, the left boundary and right boundary of the model were fixed.

2. Potential Function Model. The interatomic potentials occupy an important place in molecular dynamics simulations. Sun et al. [19] is performed to model crack propagation and calculate the interatomic potentials of $\gamma$-TiAl alloys. The total potential energy is given by

$$
E=\sum_{i} F_{i}\left(\rho_{i}\right)+\frac{1}{2} \sum_{j \neq i} \phi_{i j}\left(r_{i j}\right),
$$


where $F_{i}$ is the embedded energy as a function of the local electron density, $\rho_{i}$ is the electron density at the atom $i, \phi_{i j}$ is a pair potential interaction, and $r_{i j}$ is the distance between $i$ and $j$.

3. Simulation Process. During equilibrium, the system temperature is controlled by the method of rescaling the atom velocities. The initial velocity is the Maxwell-Boltzmann distribution. Energy minimization and conjugate gradient method are imposed to balance the system. During deformation, the system temperature is kept at $300 \mathrm{~K}$ using the Nose-Hoover thermostat in the NPT ensemble applied to the system during the loading process. Atomic trajectory is calculated via velocity - Verlet integration algorithm method. The common neighbor analysis (CNA) method [20] is used to highlight the defects, such as dislocations, stacking faults, and twinning [21]. Atomic configurations are visualized by OVITO [22].

4. Results and Discussion. When the inclination angle is $0^{\circ}$, during elastic deformation, without the consideration of the material elongation along the y direction, it can be found that the crystal structure has been not changed. This implies that the lattice bears external forces by changing the distance between atoms in the crystal structure rather than changing the crystal structure in the initial tensile stage. At the moment, the stress concentration occurs at two crack surfaces. With the increasing strain, the atoms of the crack surfaces present an out-of-order state. The atoms are out of the ideal position. After the integrity of the crystal is violated, the model presents an amorphous state. Chaotic arrangement of the atoms results in $1 / 6<112>$ Shockley dislocations and $1 / 2<110>$ full dislocations nucleation. As the energy is further increased, once more than the Peierls barrier, the dislocations will be emitted. The first dislocation is emitted at $t=86 \mathrm{ps}$. The atomic lattice rearranges, and strain energy is released. Moreover, a disordered region is formed around the crack tip, which includes $1 / 6<112>$ partial dislocations, $1 / 2<110>$ full dislocations and $1 / 6<110>$ stair-rod dislocations. Then the material undergoes plastic deformation, while dislocations are hindered by lattice resistance and slip along (111) plane. The phenomenon is consistent with the theoretical explanation that the distance is the longest in the close-packed plane and the interatomic force is the weakest. Due to boundary image force, dislocations slip towards the boundary. As the load is further increased, more atomic bonds get broken and recombined. The disordered regions become large. The crack extends by means of driving the disordered regions. During crack propagation, there are dislocations nucleation and emission in the disordered region. The dislocation slips destroy the regular stacking sequence of partial crystal atoms. The microstructure of crack tip indicates that when $t=86 \mathrm{ps}$, there are the two-layer intrinsic stacking faults with HCP structure along $(11 \overline{1})$ plane and $(1 \overline{1} 1)$ plane formed, as shown in Fig. 3a. Figure $3 b$ shows atomic configurations at $t=94 \mathrm{ps}$. It can be found that the stacking faults propagate along two close-packed surfaces. It is obvious that the growth rate along the $(11 \overline{1})$ plane is higher than along the (1) 1 ) plane. When the stacking faults propagate along (111) plane, partial dislocations $1 / 6$ $\left[\begin{array}{ll}2 & 1\end{array}\right]$ and $1 / 6\left[\begin{array}{ll}1 & 21\end{array}\right]$ are combined into $1 / 6$ [110] stair-rod dislocation since they slip along the different plane, as can be seen from Fig. 4. It is commonly known as Lomer-Cottrell lock [23], which can improve the strength to some extent.

With the expansion of the stacking faults, the void is formed at $t=97 \mathrm{ps}$ because of the fracture of atomic bonds. As the plastic deformation is further increased, the separation of atoms is enlarged and gradually forms vacancy. Finally, vacancies evolve into void. As strain is increased, the void formed the subcrack. At the moment, 1/6 [110] stair-rod dislocations disappear. The subcrack breaks away from main crack and forms the new crack tip. Then the subcrack begins to propagate toward the smaller stress concentration region.

Although the loading is homogeneous, the Brownian motion is random. So the crack extends straight along the $[100]$ direction, then along the $[\overline{1} 00]$ direction. The void appears at the crack tip at $t=103 \mathrm{ps}$, then evolves into the subcrack. During the subcrack 


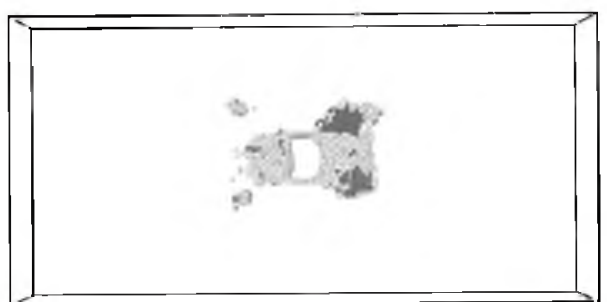

a

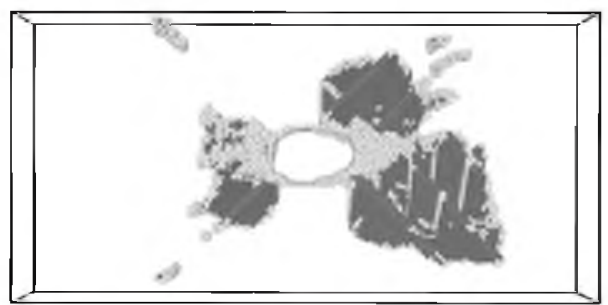

b

Fig. 3. Atomic configurations for the crack inclination angle $0^{\circ}$ at different time instants: (a) $t=86 \mathrm{ps}$; (b) $t=94$ ps.

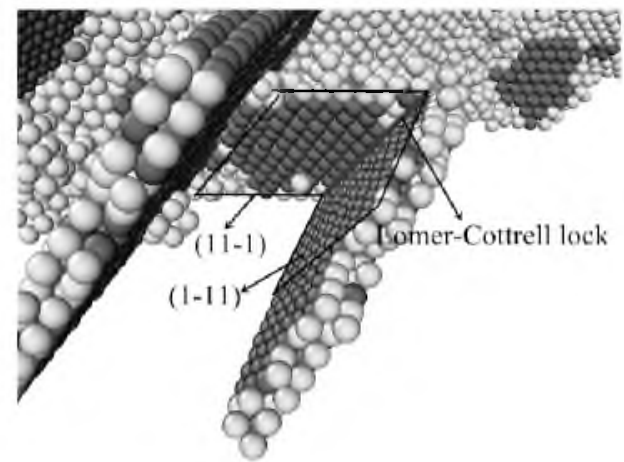

Fig. 4. The Lomer-Cottrell lock.

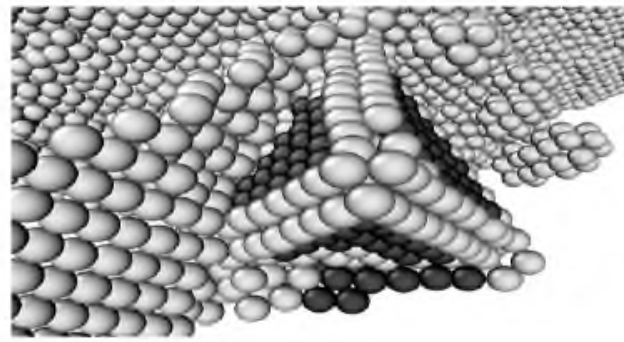

Fig. 5. Stacking fault tetrahedra.

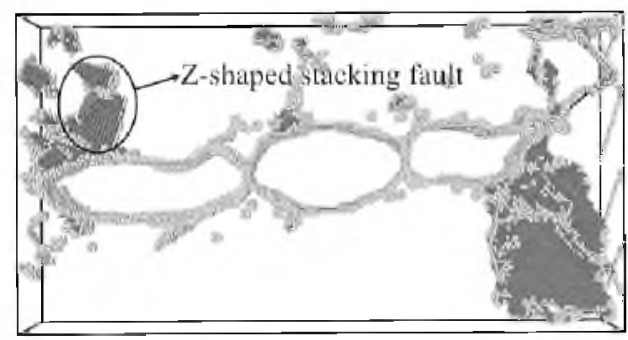

Fig. 6. Z-shaped stacking fault.

propagates, stacking fault tetrahedron (SFT) is formed, as can be seen from Fig. 5. The edges of the tetrahedron are composed of stair-rod dislocations. The surfaces of the tetrahedron are composed of stacking faults. There is the Z-shaped stacking fault formed at the subcrack tip, as can be seen from Fig. 6. The material is fully broken down at $t=292 \mathrm{ps}$. A large number of dislocations and stacking faults disappear from crack [24]. However, Z-shaped stacking fault does not completely disappear.

During crack propagation, $1 / 6[\overline{2} 11]$ and $1 / 6[2 \overline{1} 1]$ partial dislocation are combined into a new partial dislocation 1/3 [001], commonly known as the Hirth lock [25]. The stability of the dislocations is worse than $1 / 6[110]$ dislocations.

Figure 7 depicts the atomic configurations at different times when the angle is $45^{\circ}$. Because of the discontinuity of the geometry, the stress concentration appears at the crack tip. At $t=66 \mathrm{ps}$, the first dislocation is emitted from the crack tip, which results in the disordered structure at the crack tip. Therefore, a dislocation-free zone is formed in the front of the crack tip. As dislocations slip toward the boundary along the close-packed plane (111), the two-layer intrinsic stacking fault along the (111) plane is formed at the left crack tip. Observing the atomic configurations at $t=77 \mathrm{ps}$ finds that the stacking fault 
propagates along the (111) plane. With the crack propagating toward the left, the stacking faults gradually disappear. During crack propagation, the dislocations are piled up in the boundary and form a new dislocation source. When the dislocations emitted from the new dislocation source begin to slip, the two-layer intrinsic stacking faults are formed along the (111) plane. At $t=82 \mathrm{ps}$, the two-layer intrinsic stacking faults along the (111) plane are formed at the right crack tip. The stacking faults propagate along the (111) plane at $t=93 \mathrm{ps}$. As the crack propagates toward the right, the stacking faults gradually disappear. The void is formed because of disordered structure in dislocation-free zone. The void evolves into the subcrack. Eventually it converges with the main crack, then forms a new main crack. Then the new subcrack propagates and converges with the new main crack. Namely the crack propagates by means of the mechanism of sub-main crack propagation. At $t=158 \mathrm{ps}$, the material is fully broken down. Compared with $0^{\circ}$, the number of stacking faults and dislocations significantly decreases in the crystal. During the whole crack propagation, there are fewer Hirth and Lomer-Cottrell locks formed.

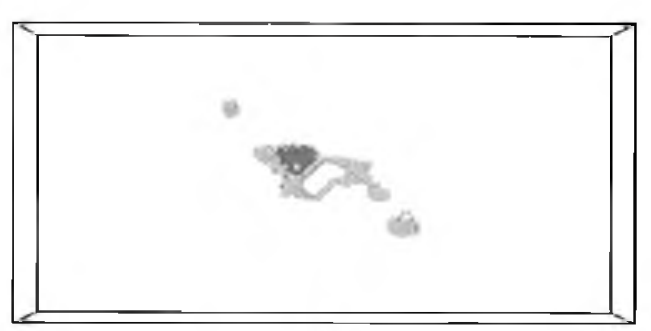

a

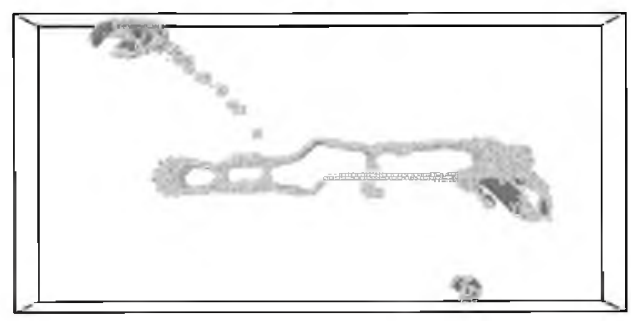

$\mathrm{c}$

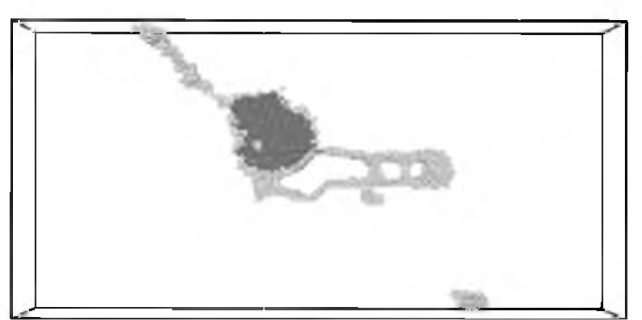

b

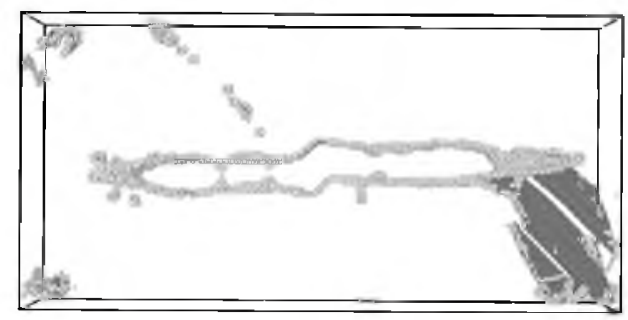

d

Fig. 7. Atomic configurations for the crack inclination angle $45^{\circ}$ at different time instants: (a) $t=70 \mathrm{ps}$; (b) $t=77 \mathrm{ps}$; (c) $t=82 \mathrm{ps}$; (d) $t=93 \mathrm{ps}$.

Figure 8 illustrate the atomic configurations at different times when the angle is $90^{\circ}$. The first dislocation is emitted from crack tip at $t=63 \mathrm{ps}$. A dislocation-free zone is formed in the front of the crack tip. The crack propagates by means of driving the dislocation-free zone. The way of crack propagation is identical with the angle $45^{\circ}$. At $t=79 \mathrm{ps}$, the two-layer intrinsic stacking faults along the (111) plane and the $(11 \overline{1})$ plane are formed at the crack tip. The stacking faults propagate along the $(\overline{1} 11)$ plane and the

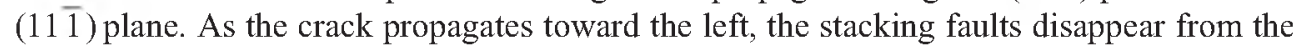
new subcrack. When the crack propagates toward the right, the dislocations are piled up in the lower right boundary, which results in the formation of the new dislocation source. When the dislocations are emitted from the new dislocation source and glide, the two-layer intrinsic stacking faults are formed along the (111) plane. As the crack further propagates toward the right, the dislocations are piled up in the upper right boundary, which results in the formation of the new dislocation source. When the dislocations glide from the dislocation source, the two-layer intrinsic stacking faults are formed along the ( $\overline{1} 11)$ plane. At $t=150 \mathrm{ps}$, the material is fully broken down. At the moment, there are only a small 


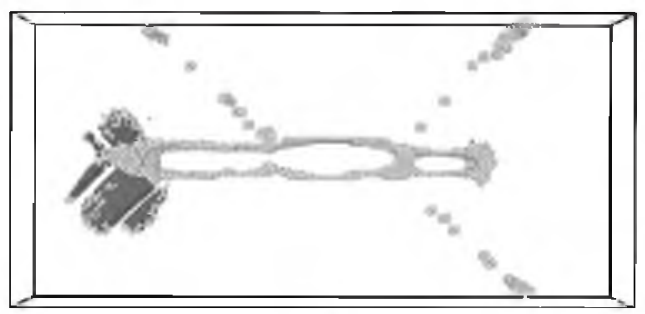

a

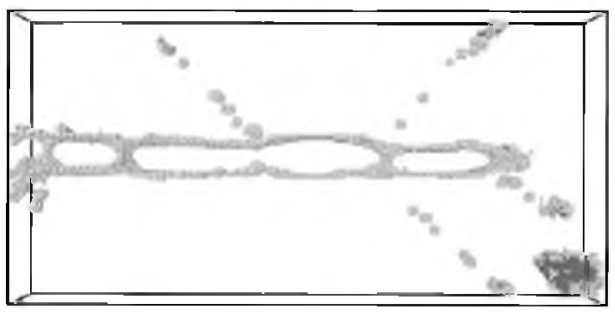

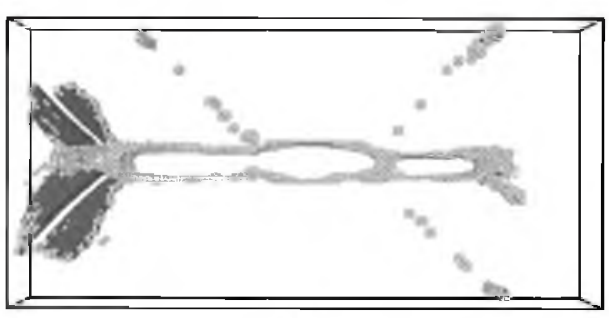

b

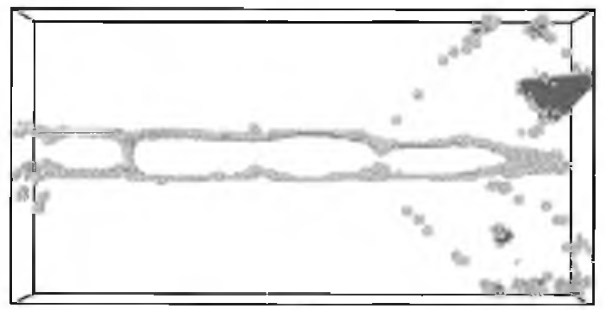

d

Fig. 8. Atomic configurations for the crack inclination angle $90^{\circ}$ at different time instants: (a) $t=79 \mathrm{ps}$; (b) $t=82 \mathrm{ps}$; (c) $t=93 \mathrm{ps}$; (d) $t=121 \mathrm{ps}$

number of dislocations without stacking fault in the crystal. During the whole crack propagation, the number of Hirth locks and Lomer-Cottrell ones is far less than $0^{\circ}$ and is identical with $45^{\circ}$.

As for the different angle between microcrack and loading direction, the stress-strain curves for crack propagation are plotted in Fig. 9. During the elastic deformation stage, the stress-strain curves are overlapped. The stresses increase almost linearly. The phenomenon is consistent with the Hooke law. It also implied that the elastic modulus is not affected by the angle. As the angles increase from 0 to $90^{\circ}$, the critical stress decreases from $9.36 \mathrm{GPa}$ at $0^{\circ}$ to $8.13 \mathrm{GPa}$ at $45^{\circ}$, and then drops from $8.13 \mathrm{GPa}$ at $45^{\circ}$ to $7.91 \mathrm{GPa}$ at $90^{\circ}$. The drop in yield stress shows that the critical stress for dislocation nucleation decreases with the angle. It also indicates that the angle effect on crack propagation weakens with the angle.

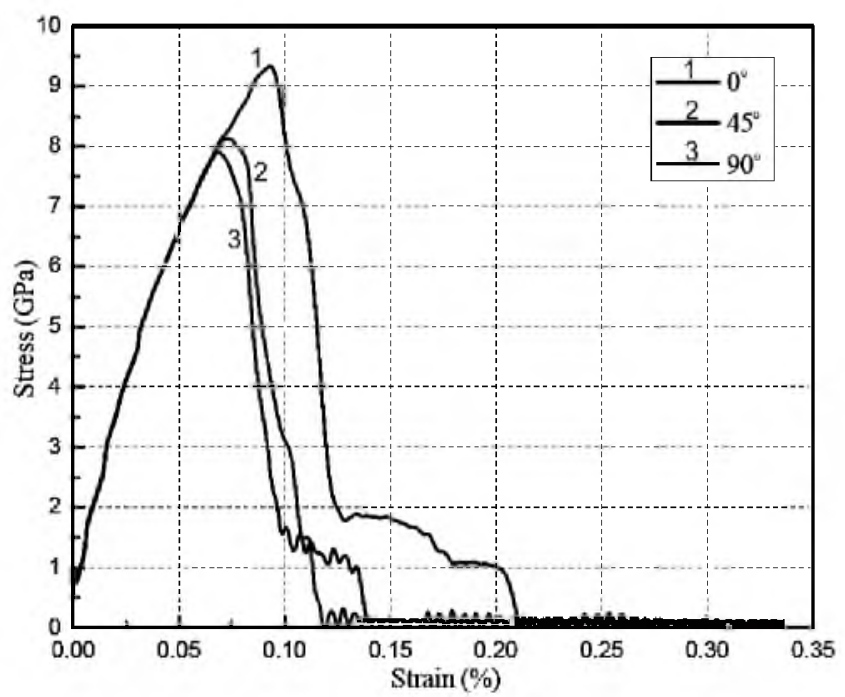

Fig. 9. Stress-strain relations for different crack inclination angles. 
As strain is further increased, the atomic bond is broken down, the stress is released. The drop in stress indicates that the material begins to enter the plastic deformation. Primarily, the model with the angle $90^{\circ}$ enters, following with $45^{\circ}$. Finally, the model with the angle $0^{\circ}$ enters. The stress for the angle $45^{\circ}$ drops to zero immediately after yielding, implying that the fracture is brittle. As for 0 and $90^{\circ}$, the stress decreases due to the rupture of atomic bond and increases again due to the formation of the new bond in the plastic deformation. Thus the stress vibrates in the stress-strain curve. Once the rate of the rupture of atomic bond exceeds the rate of formation of the new bond, the stress drops sharply until the final fracture.

Conclusions. In this study, molecular dynamics method is employed to model crack growth and investigate the mechanical properties of single-crystal $\gamma$-TiAl alloy when adopting different angle between microcrack and loading direction. According to the above analysis, the following conclusions are obtained.

1.Different angle values imply different time periods required for the first dislocation emission: larger angles correspond to shorter time periods. When the dislocations slip along the close-packed plane, intrinsic stacking faults, the Hirth and Lomer-Cottrell locks are formed, and as the angle increases, the their number decreases. In addition, the stacking fault tetrahedral appears only in the model with $0^{\circ}$.

2. With the crack expansion, fracture firstly occurs at the angle is $90^{\circ}$, and finally occurs at $0^{\circ}$.

3. The crack inclination angle has a significant influence on the crack propagation behavior. With the angle increase, the critical stress drops from 9.36 to $7.91 \mathrm{GPa}$, while the ultimate strain is also reduced from 9.4 to $6.93 \%$.

Acknowledgments. This work was financially supported by National Natural Science Foundation of Gansu Province, China (No. 148RJZA008), Research project of Gansu Province, China (No. 2014A-033) and Doctoral research fundation of Lanzhou University of Technology. Feng Ruicheng would like to thank Key Laboratory of Digital Manufacturing Technology and Application, the Ministry of Education, Lanzhou University of Technology for providing help.

1. Y. Chen, H. Niu, J. Tian, et al., "Research progress of particulates reinforced TiAl based composites," Rare Metal Mat. Eng., 40, No. 11, 2060-2064 (2011).

2. R. C. Feng, Z. Y. Rui, G. T. Zhang, et al., "Improved method of fatigue life assessment for TiAl alloys," Strength Mater., 46, No. 2, 183-189 (2014).

3. J. R. Rice, "Dislocation nucleation from a crack tip: An analysis based on the Peierls concept," J. Mech. Phys. Solids, 40, No. 2, 239-271 (1992).

4. Z. G. Wang, S. Y. Qiu, and Y. H. Wen, "Molecular dynamics simulations of the melting of nickel nanclusters," J. At. Mol. Phys., 25, No. 4, 848-852 (2008).

5. D. Terentyev, E. E. Zhurkin, and G. Bonny, "Emission of full and partial dislocations from a crack in BCC and FCC metals: An atomistic study," Comp. Mater. Sci., 55, 313-321 (2012).

6. Y. Zhou, H. Song, M. R. An, et al., "Molecular dynamics simulations of grain boundary effect on crack propagation in bicrystal magnesium," Ordnance Mater. Sci. Eng., 37, No. 1, 8-12 (2014).

7. M. Karimi, T. Roarty, and T. Kaplan, "Molecular dynamics simulations of crack propagation in Ni with defects," Model. Simul. Mater. Sc., 14, No. 8, 1409-1420 (2006).

8. X. Yan, J. G. Xu, and X. Y. Hu, "Molecular dynamics study of single-crystal Al crack growth mechanism," Ordnance Mater. Sci. Eng., 35, No. 5, 9-12 (2012). 
9. P. Guo, Molecular Dynamics Study of Single-Crystal Silicon and Aluminum the Pre-Crack Propagation, Zhengzhou University, Zhengzhou (2007).

10. H. Rafii-Tabar, H. M. Shodja, M. Darabi, and A. Dahi, "Molecular dynamics simulation of crack propagation in fcc materials containing clusters of impurities," Mech. Mater., 38, No. 3, 243-252 (2006).

11. M. H. Musazadeh and K. Dehghani, "Molecular dynamic simulation of crack propagation in nanocrystalline Ni containing different shapes and types of second phases," Comp. Mater. Sci., 50, No. 11, 3075-3079 (2011).

12. T. Tang, S. Kim, M. F. Horstemeyer, and P. Wang, "Atomistic modeling of crack growth in magnesium single-crystal," Eng. Fract. Mech., 78, No. 1, 191-201 (2011).

13. R. Fu, Z. Y. Rui, C. F. Yan, et al., "Molecular dynamics simulation of micro-crack propagation behavior in single-crystal $\gamma$-TiAl," J. Funct. Mater., 46, No. 13, 13100 13105 (2015).

14. X. J. Wang, B. Q. Zhu, and H. M. Wang, "Effects of temperature on growth of monocrystalline aluminum crack - a molecular dynamics study," J. Syst. Simul., 22, No. 2, 534-536 (2010).

15. W. P. Wu and Z. Z. Yao, "Molecular dynamics simulation of stress distribution and microstructure evolution ahead of a growing crack in single-crystal nickel," Theor. Appl. Fract. Mec., 62, 67-75 (2012).

16. G. W. Qin and S. M. Hao, "Ti-Al system intermetallic compounds," Rare Metal Mat. Eng., 24, No. 2, 1-7 (1995).

17. S. Plimpton, "Fast parallel algorithms for short-range molecular dynamics," J. Comput. Phys., 117, No. 1, 1-19 (1995).

18. Y. F. Guo and S. W. Gao, "Atomistic simulation of crack propagationand its size-dependent behavior," J. Northern Jiaotong Univ., 9, No. 4, 5-9 (2005).

19. D. Y. Sun, M. I. Mendelev, C. A. Becker, et al., "Crystal-melt interfacial free energies in hep metals: A molecular dynamics study of Mg," Phys. Rev. B, 73, No. 2, 024116 (2006).

20. D. Faken and H. Jónsson, "Systematic analysis of local atomic structure combined with 3D computer graphics," Comp. Mater. Sci., 2, No. 2, 279-286 (1994).

21. Y. G. Zheng, J. Lu, H. W. Zhang, and Z. Chen, "Strengthening and toughening by interface-mediated slip transfer reaction in nanotwinned copper," Scripta Mater., 60. No. 7, 508-511 (2009).

22. A. Stukowski, "Visualization and analysis of atomistic simulation data with OVITO The open visualization tool," Model. Simul. Mater. Sc., 18, No. 1, 015012 (2010).

23. S. Z. Chavoshi and X. Luo, "Molecular dynamics simulation study of deformation mechanisms in 3C-SiC during nanometric cutting at elevated temperatures," Mater. Sci. Eng. A, 654, 400-417 (2016).

24. Y. F. Fu, Molecular Dynamics studies of Plastic Deformation of Nanotwinned Copper with Embedded Cracks, Dalian University of Technology, Dalian (2014).

25. T. Paulauskas, C. Buurma, E. Colegrove, et al., "Atomic scale study of polar Lomer-Cottrell and Hirth lock dislocation cores in CdTe," Acta Crystallogr. A, 70, No. 6, 524-531 (2014). 